\section{El uso de metodologías altamente competenciales de Educación Física para el aprendizaje de la orientación espacial en Primaria}

\author{
The use of high competency-based learning methodologies \\ of Physical Education to promote the spatial orientation in \\ Primary school
}

\section{MÍRIAM SEGURA}

Depart. de Pedagogía, Universidad Rovira y Virgili

CARME JULIÀ

Depart. de Ingeniería Informática y Matemáticas, Universidad Rovira y Virgili

\section{RESUMEN}

Esta investigación propone la Educación Física para trabajar la orientación espacial. La metodología propuesta consiste en usar dos estilos altamente competenciales: estilo de producción convergente y estilo de producción divergente. En estos, los alumnos deben aprender a solucionar los problemas planteados y no limitarse a imitar patrones motores de sus compañeros o maestros. El maestro asume el papel de guía. Presentamos una experiencia llevada a cabo en un grupo experimental y un grupo control de $5^{\circ}$ de Primaria. Los resultados muestran una mejora más notable de la orientación espacial en el grupo experimental. Además, se evidencia un elevado grado de motivación por parte de estos alumnos durante las sesiones de Educación Física. Finalmente, se observa que la metodología utilizada y la organización del alumnado facilitan la adquisición de contenidos clave actitudinales. Estos resultados permiten concluir que se podría usar una propuesta similar para trabajar diferentes áreas experimentales de forma competencial y motivadora.

\section{ABSTRACT}

This investigation proposes to use the Physical Education to promote the spatial ability. The proposed methodology uses two very competency-based learning styles: convergent productive style and divergent productive style. In these styles, students must learn to solve problems raised in class and not just imitate gestures or motor patterns of their classmates or teachers. The teacher assumes the role of guide. We present a practical experience carried out in an experimental group and a control group of 5th Primary school students. Results manifest the notable improvement of the spatial ability in the experimental group case. Additionally, it is evident the high level of motivation shown by these students during the sessions of Physical Education. Finally, it is observed that the methodology used and the organization of students have facilitated the acquisition of key attitudinal contents. These results allow to conclude that a similar proposal could be used to study different experimental areas in a competency-based and motivating way.
Recibido: 17/07/2017

Aceptado: 08/02/2018

PALABRAS CLAVES

Educación Física, orientación espacial, metodología competencial, motivación, Educación Primaria.

\section{KEYWORDS}

Physical education, Spatial Ability, Competency-based Learning, Motivation, Primary Education. 


\section{INTRODUCCIÓN}

La Educación Física es un área privilegiada desde la cual enseñar y educar a nuestros alumnos. Cada asignatura, debido a la especificidad de sus contenidos, dispone de una didáctica concreta (Sebastiani, 2003). El carácter lúdico de la Educación Física, los aspectos vivenciales que en ella tienen cabida y la implicación del alumnado a nivel físico, psíquico y social facilita que se trate de una asignatura bien considerada entre los alumnos.

A diferencia de otras áreas tradicionalmente centradas en el trabajo de contenidos, en Educación Física no es nuevo el hecho de trabajar de forma competencial (Blázquez, 2013). En general, se planifican y evalúan competencias en lugar de contenidos. En Educación Física la demanda constante de actividad, la globalidad desde la que se trabaja, la exigencia continua de mostrar las habilidades y destrezas que uno posee, la imposibilidad de disimular los errores y los progresos, por no hablar del espacio, material o agrupación de los alumnos hacen que disponga de unas características propias, muy distintas de las que presentan las asignaturas del aula ordinaria. Estos matices generan la necesidad de secuenciar, diseñar y evaluar por competencias, entendiéndose éstas como la capacidad que tiene una persona para utilizar sus conocimientos y habilidades para resolver un problema o una situación de la vida cotidiana.

Al mismo tiempo, el currículum abierto en cuanto a contenidos permite diseñar experiencias de aprendizaje práctico para trabajar contenidos de distintas áreas de una forma más competencial y motivadora. De hecho, son varios los estudios que demuestran la capacidad de la Educación Física de trabajar distintos contenidos relacionados con otras áreas como matemáticas o ciencias naturales, sociales, lengua, etc. (e.g., Sallan, 2002; Bocanegra y Villanueva, 2003).

Por otro lado, la opinión sobre la asignatura de matemáticas suele ser opuesta a la comentada en el caso de Educación Física. Díaz (2010) propone aprovechar el carácter lúdico-formativo de la Educación Física y su aceptación, en general, entre los alumnos como el puente idóneo para el desarrollo transversal de las matemáticas. En su artículo, Díaz presenta propuestas de enseñanza-aprendizaje para trabajar los diferentes bloques de contenido de matemáticas: número y operaciones, medida, estimación y cálculo de magnitudes, geometría y tratamiento de la información, azar y probabilidad.

Teniendo en cuenta las características ya comentadas de la asignatura de Educación Física, esta investigación se centra en potenciar la orientación espacial de los alumnos de Primaria, un contenido que no siempre se enseña en las clases de matemáticas.

Existen diversas definiciones para el término habilidades espaciales. Algunas de ellas contienen de forma implícita la orientación espacial, razonamiento espacial o la visualización. Sutton y Williams (2007) definen las habilidades espaciales como aquellas que se necesitan para realizar rotaciones mentales de objetos, describir y entender cómo se ven los objetos desde diferentes ángulos y entender cómo se relacionan los objetos entre ellos en el espacio. Sutton, Heathcote y Bore (2005) mencionan que las habilidades espaciales permiten extraer información sobre propiedades en tres dimensiones (3D) a partir de representaciones de éstas en dos dimensiones (2D). En (Newcombe, 2010), se usa el término pensamiento espacial (spatial thinking) y se define como la habilidad de localizar los objetos, sus formas, sus relaciones entre ellos y los recorridos que toman cuando se mueven. Newcombe comenta la importancia de trabajar la orientación espacial para mejorar el aprendizaje de las matemáticas y las ciencias, entre otras áreas.

En 2000, el National Council of Teachers of Mathematics (NCTM) marcó unos objetivos y recomendaciones para la educación de las matemáticas desde nivel preescolar hasta la educación secundaria. En el caso concreto 
de Geometría, los autores proponen que los alumnos analicen las características de las formas y que construyan argumentos matemáticos sobre las relaciones geométricas, así como el uso de la visualización, el razonamiento espacial y el modelaje geométrico para resolver problemas. Además de las recomendaciones del NCTM sobre introducir la visualización y las habilidades espaciales en Primaria, existen varios autores que insisten en la importancia de adquirir estas habilidades para mejorar resultados en áreas como ciencias, tecnología o ingeniería (ver por ejemplo: Sorby, 2009; Sutton y Williams, 2007; Verner, 2004).

En el caso de la Educación Física, la orientación deportiva es definida por Morales y Guzmán (2000) citado por Valero y col. (2010) como la realización de un recorrido por diferentes lugares, de una zona delimitada, conocidos o no, dibujado en un mapa, pasando por unos controles fijados en el terreno en un orden establecido. Aunque los trabajos que se acaban de citar usan diferentes términos o definiciones para referirse a las habilidades espaciales, lo importante es que todos proponen potenciar estas habilidades (o alguna de ellas) en educación Primaria.

El principal objetivo de este trabajo de investigación es el de potenciar la orientación espacial a través de la Educación Física. Para conseguir este objetivo general, se plantean los siguientes objetivos específicos:

- Mostrar que es posible trabajar contenidos de otras áreas instrumentales, usando metodologías o juegos propios de Educación Física.

- Estudiar la motivación de los alumnos cuando asisten a clases que siguen una metodología altamente competencial.

- Remarcar e interiorizar la importancia de potenciar el trabajo cooperativo.

En cuanto a la metodología, se utilizan dos estilos de enseñanza-aprendizaje altamente competenciales: el estilo de producción convergente y el estilo de producción divergente (Zabala, 2014). Éstos son muy diferentes de los que se usan habitualmente en Educación Física: el descubrimiento guiado y la asignación de tareas, ambas menos competenciales. Los estilos de producción convergente y divergente son estilos, como su nombre indica, de producción, eso significa que, a diferencia de los estilos de reproducción (por ejemplo asignación de tareas) los alumnos deben aprender a encontrar soluciones a los problemas planteados en clase y no limitarse a copiar, imitar, repetir los gestos o patrones motores de sus compañeros o maestros. A lo largo de este proceso cognitivo, motriz, afectivo y de toma de decisiones el maestro es un guía, facilita ayuda en el proceso, pero ni propone ni inhabilita soluciones, son los alumnos los que deben hacer el aprendizaje y llegar a conclusiones o propuestas finales trabajando en equipo de forma cooperativa. La metodología de enseñanza-aprendizaje escogida permite establecer un adecuado engranaje de contenidos y áreas.

Para el desarrollo de esta propuesta didáctica, se propone organizar la clase en grupos pequeños, para intentar fomentar las capacidades comunicativas, el trabajo cooperativo y la autonomía de los alumnos. De esta forma, se trabaja también la resolución de problemas de una forma más lúdica.

El resto del artículo está estructurado de la siguiente forma: primero, se presenta la metodología utilizada durante la investigación. A continuación, se muestran y analizan los resultados obtenidos, teniendo en cuenta los datos que proporcionan los diferentes instrumentos de control que se han usado en esta investigación. Se finaliza el artículo con unas conclusiones y unas implicaciones didácticas. 


\section{METODOLOGÍA}

La metodología que se siguió en la investigación es de carácter cuantitativo, descriptiva-interpretativa, basada en la secuencia pretest-proceso de enseñanza-postest y trabajando con grupos experimental y control.

\subsection{Población y muestra}

La propuesta se llevó a cabo en una escuela concertada. En concreto, la práctica está orientada para alumnos de $5^{\circ}$ de Primaria. Aprovechando que la escuela es de dos líneas, la clase de $5^{\circ} \mathrm{A}$, formada por 21 alumnos, se consideró como grupo control (GC) y la clase de $5^{\circ} \mathrm{B}$, formada por 24 alumnos, se tomó como grupo experimental (GE).

\subsection{Propuesta de enseñanza}

El experimento de enseñanza se inició aplicando un pre-test a todos los alumnos de $5^{\circ}$ de Primaria (GC y GE) para identificar el nivel de partida respeto a la orientación espacial. Posteriormente se realizaron cinco sesiones de Educación Física únicamente al GE. Durante las sesiones de clase, los alumnos de este grupo completaron un instrumento de autoevaluación. Finalmente, se administran a todos los alumnos los cuestionarios para recoger los resultados finales y comprobar si había diferencia entre los dos grupos. En el caso del GE, se les pasó también un test para evaluar su grado de motivación durante las sesiones de la secuencia experimental. La segunda autora del artículo pasó los test y la primera impartió las sesiones de Educación Física.

\subsection{Diseño de la secuencia experimental}

La secuencia didáctica, llamada Los aprendices de detective, se diseñó y temporizó conjuntamente con el maestro de Educación Física del centro. La selección y el diseño de las actividades de la secuencia didáctica se basaron en el currículum de Primaria de la comunidad autónoma donde está ubicado el centro educativo, considerando los contenidos clave de la Educación Física de tercer ciclo: control y conciencia corporal, lateralidad, orientación espacio temporal, resolución de situaciones motrices y habilidades motoras básicas.

Como se ha comentado anteriormente, se organizó la clase en grupos de 4/5 alumnos y se usaron dos estilos de enseñanza-aprendizaje altamente competenciales: el estilo de producción convergente y el de producción divergente. En ambos estilos se les plantea a los alumnos una situación problema a resolver. Los alumnos, utilizando sus competencias básicas (lingüística, social y ciudadana, interacción con el mundo físico, entre otras), deben, en el primero (producción convergente), escoger entre todo el grupo la mejor solución. En el estilo de producción divergente son válidas distintas soluciones al mismo problema y cada alumno puede defender la suya y compartirla con los demás para valorar si es adecuada o no.

La metodología utilizada y la organización de los alumnos en las distintas actividades también facilita la adquisición de contenidos clave actitudinales: trabajo en equipo, cooperación, respeto, entre otros. La Tabla 1 muestra las sesiones de la secuencia diseñada e implementada en esta investigación. 
SESIÓN 1: LOS ARQUITECTOS Y LOS ESPÍRITUS MÓvILES

Organización social: Gran Grupo - Grupos de 3

\section{Actividades}

- Presentación y explicación de la secuencia.

- Bolas de dragón: encontrar las pelotas de espuma pequeñas escondidas por el maestro. Variante: las esconden la mitad de los alumnos y el resto las encuentra.

- En pequeños grupos, dibujar un plano de la pista.

- Situar diferentes objetos de EF en la pista. Los alumnos deberán situarlos en su plano.

- Un alumno mueve un objeto de la pista. El resto deben averiguar qué objeto se ha movido y dibujarlo de nuevo en el plano.

- Explicar verbalmente el proceso que han seguido para dibujar el plano y los objetos.

\section{Sesión 2: Camuflaje musical}

Organización Social: Dos grupos - Grupos de 5 - Gran grupo

\section{ACTIVIDADES}

\section{MAterial}

Pelotas de espu- Gimnasio y pista ma, conos, aros, deportiva cuerdas, hojas de papel, colores $\mathrm{y}$ lápices

\section{ESPACIO}

- Los contrabandistas con macarrones: dos equipos (contrabandistas / policía aduana). Hacer contrabando de macarrones. Cada contrabandista sólo puede ser parado por dos poliMaterial Espacio cías que deberán intentar encontrarle el macarrón utilizando las manos. Sólo una pieza por viaje.

- La cacería de los tesoros: los alumnos se organizarán en pequeños grupos. Cada grupo dispondrá de un plano con 10 marcas. Deberán localizarlas y anotarse la palabra encontrada.

- Utilizar el máximo número de palabras encontradas para cantar un máximo de dos canciones. Gana el equipo que utiliza más en un contexto correcto. 


\section{Organización social: Grupos de 5}

ACTIVIDADES

- En pequeños grupos, leer un texto y seguir las indicaciones para llegar a un lugar concreto.

- Una vez allí, dibujar el recorrido que han seguido para llegar desde el lugar de salida al punto final. Ocultar cinco objetos por la zona y marcarlos en el mapa. Volver al punto de salida.

- Intercambiar los planos entre los grupos. Ir a buscar los objetos escondidos por el otro equipo.

- Explicar cómo y dónde han encontrado los diferentes objetos escondidos.

\section{Material}

Indicaciones critas, objetos pequeños y variados para ser escondidos, hojas de papel, colores $\mathrm{y}$ lápices

SESIÓN 4: LOS DETECTIVES CONSTRUCTORES

Organización SOCIAL: Grupos dE 5

\section{ACtividades}

- En grupos de 5 alumnos, interpretar unas pistas para localizar dónde está escondida una imagen. Cada grupo inicia la carrera en un punto diferente.

- Cuando encuentran una, la memorizan, vuelven al punto inicial y deben construir y/o dibujar aquello observado. Una vez realizado correctamente, recibirán la siguiente pista.

\section{Material Espacio}

Pistas de locali- Patios exteriores, zación, imágenes, huerto y bosque piezas de construcción, hojas de papel, colores $y$ lápices

- Dibujar el mapa y ubicar todas las imágenes encontradas.

Sesión 5: Testimonios ocultos

\section{ORgANIZACIÓN SOCIAL: GRUPOS DE 5}

\section{ACtividades}

- Orientación con relieves: Repartir a cada grupo de 5 alumnos un mapa en el que habrá marcados 5 puntos. En el punto número 1 estará escondido un testigo. El primer relevista, cuando lo encuentre debe ir a esconderlo en el punto número 2 y volver al inicio para que salga el compañero siguiente. Así sucesivamente hasta encontrarlos todos.

- Creación de una carrera de relevos orientada: cada grupo de alumnos elige una zona del patio. En ella, dibujan el plano y marcan 5 puntos. En el primero, esconden un testigo. Intercambio de mapas entre los diferentes equipos para realizar el recorrido. El equipo que finaliza antes es el ganador.

\section{Material Espacio}

Relevos, mapas, Patios exteriores, hojas de papel, co- huerto y bosque lores y lápices 


\subsubsection{Ejemplo de actividad de la sesión 1}

Se propone a los alumnos dibujar un mapa del patio. Disponen de 15 minutos y del material necesario para dibujarlo. La Figura 1 muestra un ejemplo de producción de un grupo de alumnos.

\section{Figura 1}

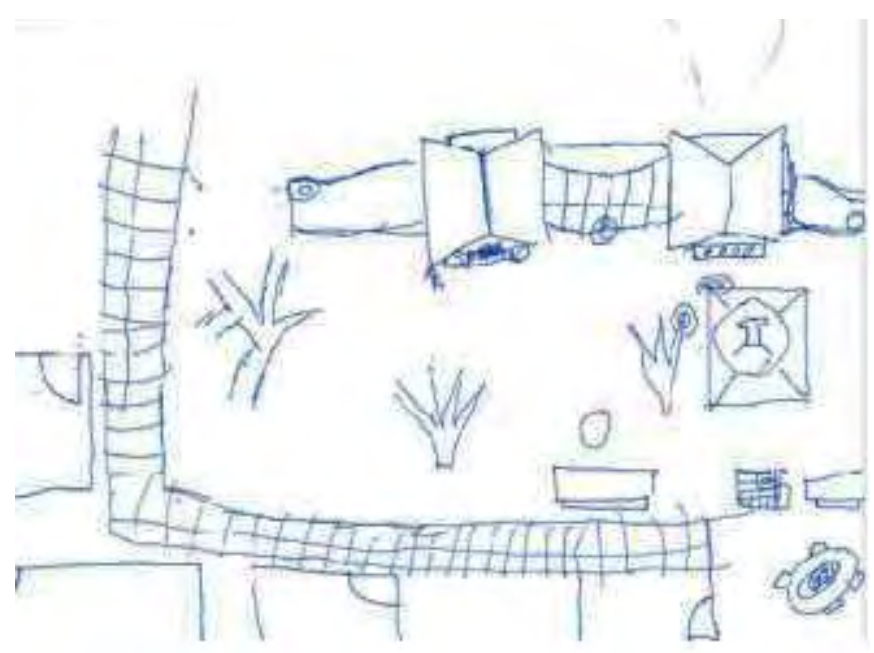

\subsubsection{Ejemplo de actividad de la sesión 2}

La cacería de los tesoros. Los alumnos disponen de un mapa como el de la Figura 2 y deben localizar 10 objetos que aparecen señalados allí. Los objetos han sido previamente escondidos por el maestro.

\section{Figura 2}

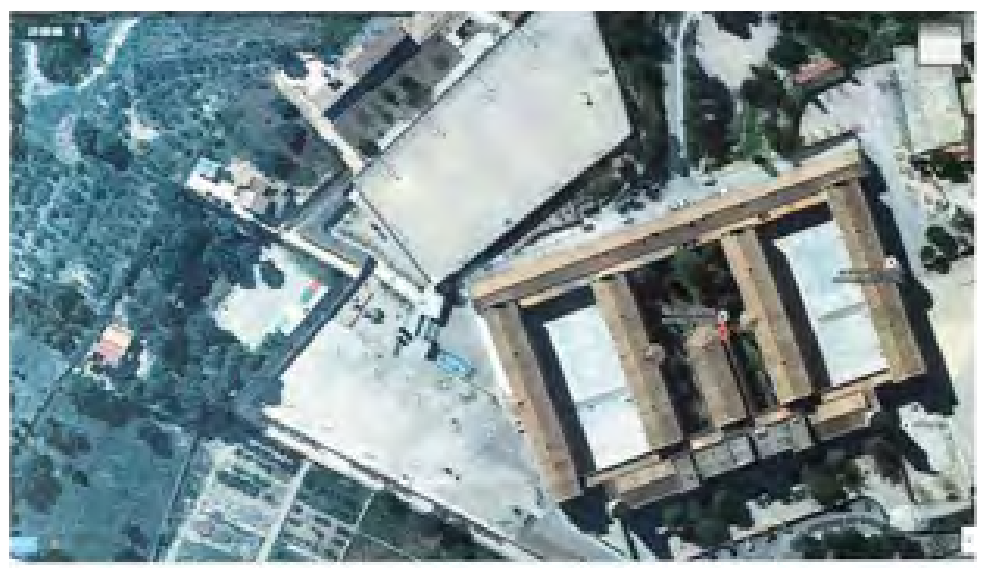




\subsubsection{Ejemplo de actividad de la sesión 3}

Lectura de texto y seguir las indicaciones para finalizar un recorrido escrito. Cada grupo dispone de un recorrido distinto.

\section{Recorrido 6}

Sitúate enfrente de la fuente y da veintidós pasos en dirección al colegio. Después realiza veintinueve pasos a la izquierda y cuatro a la derecha. Anda nueve pasos más en dirección a portería y pásala por debajo. Recorre cuarenta pasos más en línea recta y llegarás al destino.

\subsubsection{Ejemplo de actividad de la sesión 4}

Localizar distintos puntos señalados en un mapa de la escuela (ver Figura 3). Cuando los encuentran, memorizar la figura en tres dimensiones allí escondida (ver ejemplos en la Figura 4) e ir a reproducirla en el punto de partida.

Figura 3

Mapa con los distintos puntos donde deben dirigirse los alumnos (sesión 4)

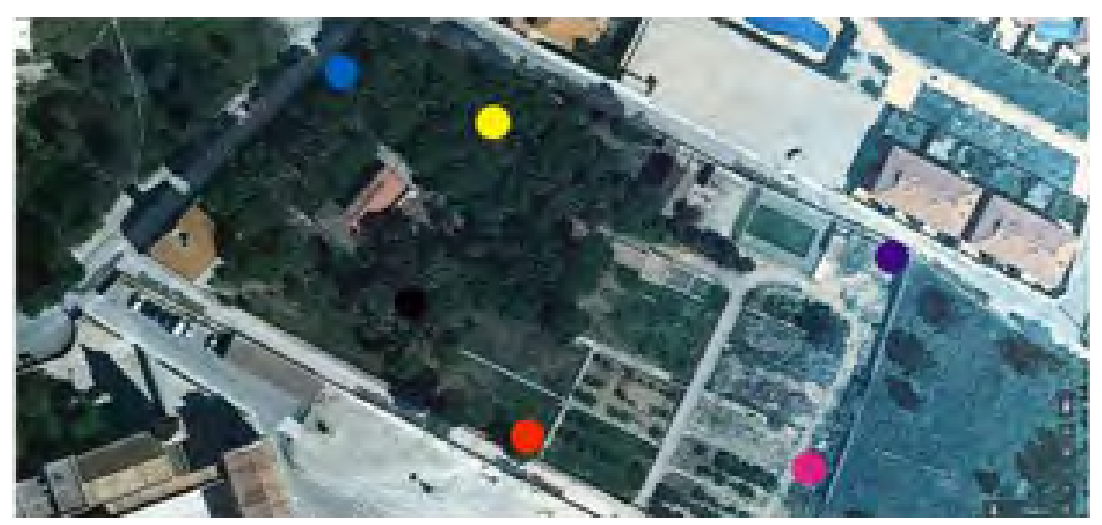


Figura 4

Ejemplos de figuras que los alumnos deberán memorizary reproducir (sesión 4)

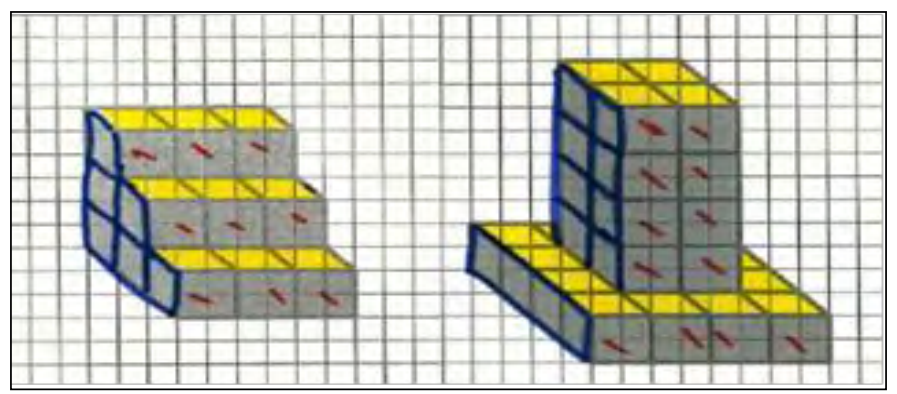

\subsubsection{Ejemplo de actividad de la sesión 5}

Diseño de un mapa de la zona en la que han realizado la carrera por equipos. La Figura 5 muestra un ejemplo de producción de un grupo de alumnos.

\section{Figura 5}

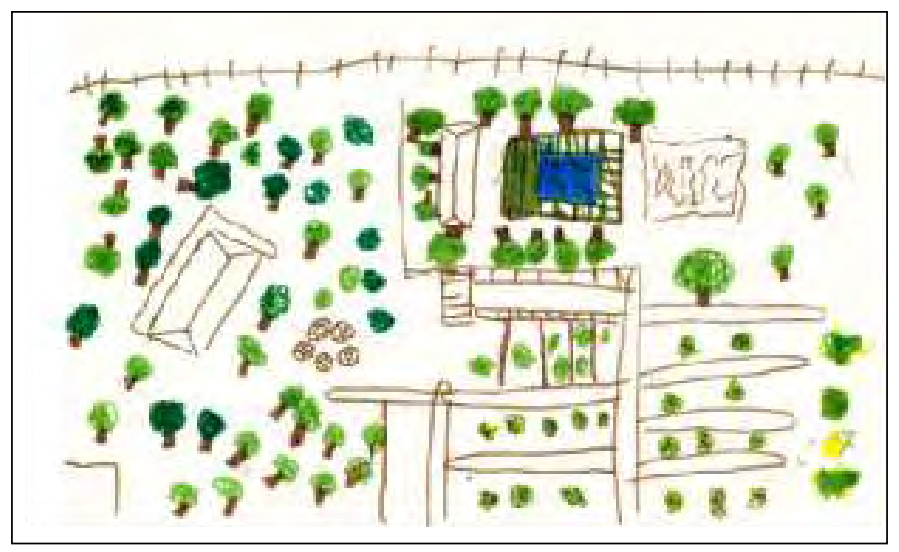

\subsection{Instrumentos de control}

En esta investigación se han utilizado distintos instrumentos para la recogida de datos: ficha de autoevaluación, test de motivación y test de orientación espacial. 


\section{Autoevaluación}

Se ha diseñado una ficha de autoevaluación para enseñar a los alumnos a reflexionar sobre su propio rendimiento y su actitud frente a las actividades realizadas y a los compañeros. En ningún caso se trata de una ficha para calificar al alumno, sino que la idea es que éste sea sincero y se dé cuenta de los aspectos que debe mejorar y cómo puede hacerlo. Este instrumento de control permite al mismo tiempo trabajar la competencia de aprender a aprender.

Se explicó a los alumnos cómo debían rellenar la ficha y se leyeron cada uno de los indicadores para constatar que habían sido entendidos. Al terminar cada sesión, los alumnos debían pintar en la celda de la derecha el nivel de acuerdo que mostraban con los dos indicadores que hacen referencia a dicha sesión. La Tabla 2 muestra la ficha de autoevaluación.

\section{Tabla 2}

Ficha de autoevaluación

$\begin{array}{ll}\text { LOS APRENDICES DE DE- } & \text { Nombre: } \\ \text { TECTIVE } & \text { Poco ________ Mucho }\end{array}$

Los arquitectos y los espíritus He ayudado a mis compañeros en el diseño del mapa. móviles He observado algún objeto que se ha movido y lo he situado en el mapa.

Camuflaje musical

He colaborado con los compañeros para encontrar las marcas.

He participado activamente en la elaboración y representación final.

Recolectores de setas

He sido capaz de dibujar, con la ayuda de los compañeros el recorrido.

He localizado algunos de los objetos escondidos por los otros equipos.

He memorizado y construido correctamente algunas figuras.

Los detectives constructores

He ayudado a los compañeros a dibujar el mapa y marcar los objetos.

He finalizado con éxito la carrera de relevos diseñada por la maestra.

Los testimonios ocultos

He participado en la creación de un nuevo recorrido de carrera.

Escala: Poco hasta mucho. Pintar el cuadro.

Observaciones - comentarios: 


\section{Test de motivación}

Se usó el Course Interest Survey (CIS) para cuantificar las reacciones de los alumnos respecto a la metodología usada en clase (Keller, 2006). Este cuestionario fue diseñado según la teoría de un modelo específico de la motivación del alumno: el modelo ARCS (Keller, 1987). Concretamente, el CIS se utiliza para medir cuatro factores de motivación: atención, relevancia, confianza y satisfacción. El objetivo de este instrumento es averiguar la motivación de los alumnos respecto a un curso que se ha impartido siguiendo una metodología nueva para ellos. Aunque según Keller el cuestionario es válido para cualquier edad, hay que tener en cuenta que para niveles de educación primaria, algunas de las preguntas pueden resultar difíciles de entender. Para este trabajo, se tradujo el cuestionario original y se adaptó alguna pregunta a la realidad concreta, tal y como se aconseja en (Keller, 2006).

El CIS consiste en 34 preguntas o afirmaciones que los alumnos tienen que puntuar entre 1 (no cierta) y 5 (muy cierta). El hecho de que algunas de las preguntas estén formuladas de forma negativa, provocó alguna confusión. El cuestionario está formado por preguntas referidas a los cuatro factores mencionados anteriormente: 8 referentes a la atención, 9 a la relevancia, 8 a la confianza y 9 a la satisfacción. Las preguntas de los factores se van intercalando a lo largo del cuestionario.

\section{Test de orientación espacial}

Tal y como se comenta en (Julià y Antolí, 2016), existe una amplia colección de instrumentos para evaluar la orientación espacial. Lamentablemente, la mayor parte de ellos están diseñados para alumnos de secundaria (más de 12 años). Recordemos que en este trabajo, los alumnos tienen 11 años. Al final, se han escogido dos de los test que se usan en (Julià y Antolí, 2016). El primero, el Paper Folding Test, está basado en la propuesta de (Bakker, 2008), donde el autor analiza diferentes test para evaluar las habilidades espaciales de niños de 11 años. El segundo, el Perspective Taking/Spatial Orientation Test (Hegarty y Waller, 2004), fue seleccionado de la Spatial Intelligence and Learning Center ${ }^{1}$, una web que recoge tests para evaluar habilidades espaciales.

El primer test (sub-test 1) cuenta con 2 partes, de 10 preguntas cada una. Estas dos partes configuran el pre y el post-test respectivamente. El segundo test (sub-test 2), lo configuran 12 preguntas que se han separado en 6 para el pre-test y 6 para el post-test. A continuación, se muestra un ejemplo de pregunta de cada uno de los sub-test.

\section{Paper Folding Test}

Los alumnos tienen que imaginar cómo se dobla y desdobla una hoja de papel. A la izquierda de cada pregunta se dan las instrucciones de cómo se ha doblado la hoja de papel (ver Figura 6). Después, se marca un agujero en el papel. Una vez desplegado el papel, hay que escoger cuál de las figuras de la izquierda corresponde al papel original de la derecha (en el ejemplo que muestra la Figura 6, la respuesta correcta es la C). 


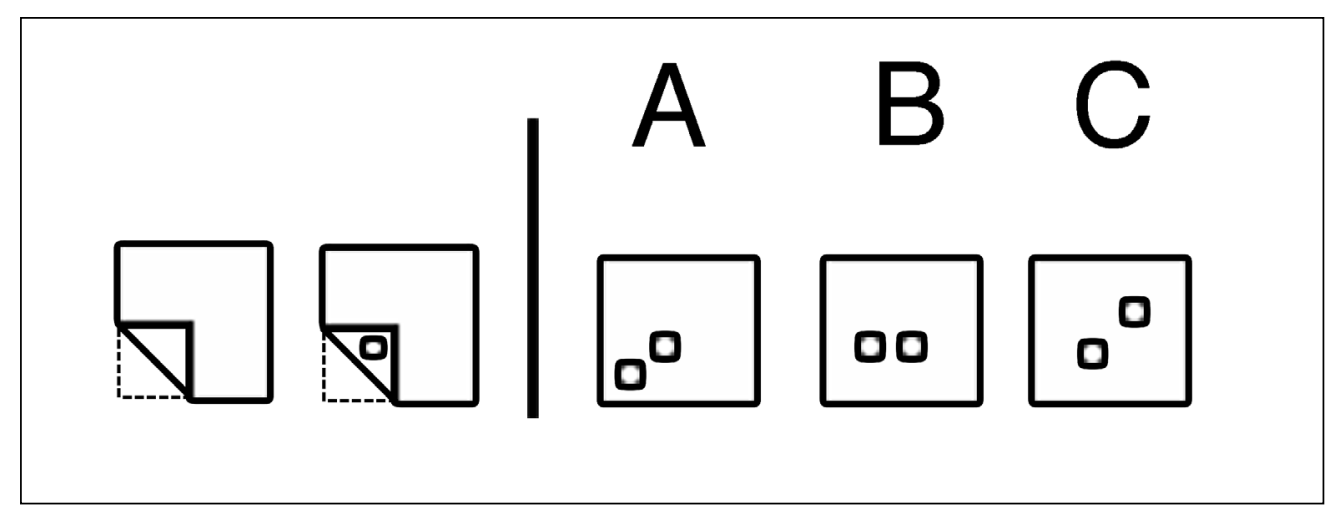

\section{Perspective Taking/Spatial Orientation Test}

Este test requiere visualizar diferentes perspectivas y orientaciones de objetos en el espacio. La Figura 7 muestra un ejemplo. Siempre hay los mismos objetos en la parte superior de la imagen. En el medio, se proporciona información sobre la posición del alumno. En la parte inferior, el alumno tiene que transformar la información que se le proporciona en un esquema. En el ejemplo que muestra la Figura 7, la línea discontinua corresponde a la respuesta esperada.

Es necesario remarcar que ninguna de las actividades que aparecen en estos test se llevó a cabo durante las sesiones de Educación Física de la secuencia didáctica que se propone en esta investigación.

Los alumnos dispusieron de 6 minutos para completar las 10 preguntas que componen el sub-test 1 y 5 minutos para completar las 6 preguntas que forman el sub-test 2 .

\section{RESULTADOS Y ANÁLISIS}

\section{1. Resultados autoevaluación}

La escala de estimación consta de 10 indicadores que son valorados por los propios alumnos del GE pintando una proporción de la celda lateral. De entre los indicadores de consecución más individuales (b, f, g, i), los que obtuvieron mejor valoración fueron los de observar y localizar objetos en el mapa (b, f). Casi el 90\% de los alumnos pintaron entera la celda. Respeto a la memorización y construcción de figuras, el intervalo de percepción de éxito se situó alrededor del 70\%.

El resto de indicadores (a, c, d, e, h, j), relativos al trabajo en equipo y a la cooperación conjunta para alcanzar un objetivo común (producción convergente-divergente) obtuvieron valores altos en la Tabla. La observación 
directa de la escalera muestra un claro acercamiento en la mayoría del alumnado al valor mucho, habiendo tres alumnos que, en general, consideraron que su aportación al grupo cooperativo y a la tarea había sido media, mejorando en las dos últimas sesiones.

Estos resultados indican que los alumnos entendieron el proceso colaborativo, participaron de él y consideraron relevante su aportación al grupo, siendo éste uno de los objetivos de la investigación.

\section{Figura 7}

\section{Perspective Taking/Spatial Orientation Test}

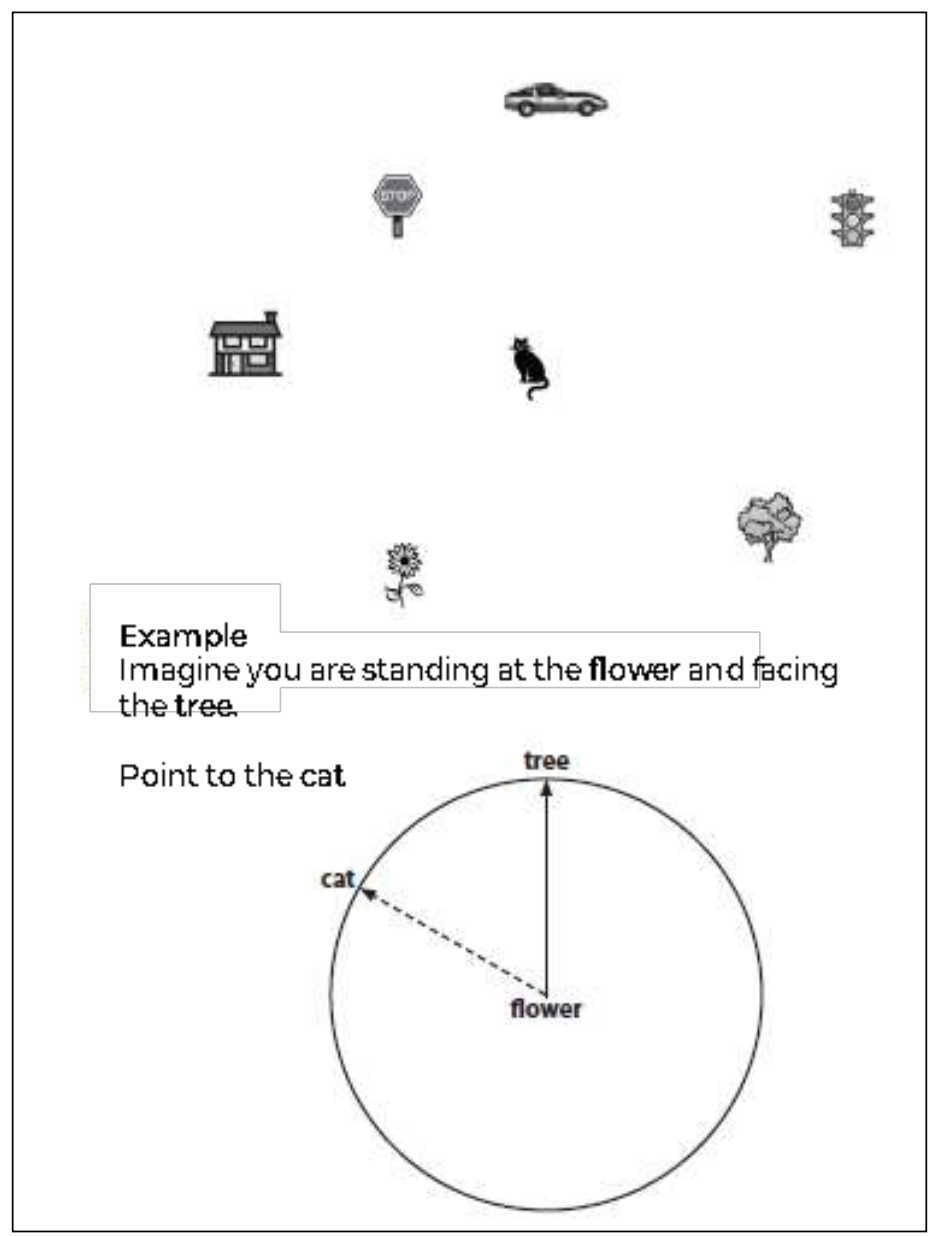

\section{2. Resultados motivación}

El test consta de 33 preguntas que se valoran entre 1 y 5 . Se eliminó una pregunta del test, porque hacía referencia a la evaluación de la actividad y no se les había proporcionado ninguna evaluación. Así, las posibles puntuaciones están entre el valor mínimo y máximo de 33 y 165, respectivamente. La Figura 8 muestra los valores que cada alumno obtuvo en el test de motivación (suma de las puntuaciones que cada alumno dio a cada una de las preguntas del test). En particular, la figura poligonal contiene los datos entre el primer y tercer cuartil (la mediana se representa por una línea horizontal en la región más estrecha). 
La media de las puntuaciones obtenidas en el test de motivación fue de 122.85 , con valores entre 73 y 149 . Nótese en la Figura 8 que el 73 es considerado un outlier (está marcado con una cruz roja), ya que la siguiente puntuación es 103, un número mucho mayor. Estos resultados permiten deducir que la mayoría de los alumnos se sintió motivado en las clases.

\section{Figura 8}

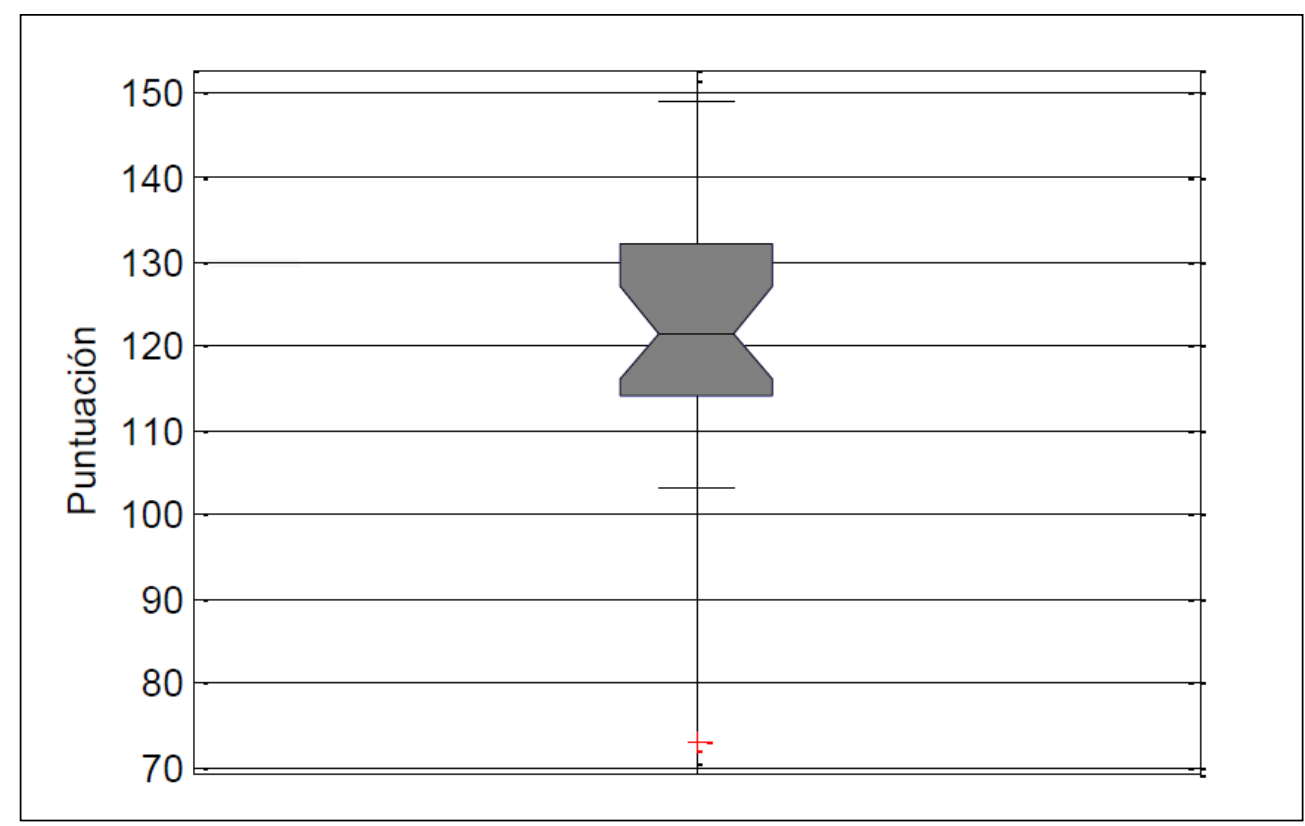

La Tabla 3 muestra la media y desviación estándar de los cuatro factores que se consideran en el modelo ARCS. Se puede observar que la satisfacción es el factor que obtiene máxima puntuación, con una media de 3.93 (valores entre 1 y 5). Este resultado indica que los alumnos están satisfechos con la metodología usada durante las sesiones de Educación Física. Los otros tres factores también obtienen puntuaciones altas, con medias de 3.69, 3.71 y 3.53 para la atención, relevancia y confianza, respectivamente. Estos resultados indican que los alumnos siguieron con atención las sesiones, que las actividades coincidieron con los objetivos de aprendizaje de los alumnos y que la mayoría de los alumnos se sintieron seguros aprendiendo con esta metodología tan competencial. En resumen, las puntuaciones obtenidas permiten pensar que la mayoría de los alumnos estuvieron motivados durante las sesiones altamente competenciales de Educación Física propuestas en este trabajo. 


\begin{tabular}{lcc} 
FActor & Media & SD \\
\hline Atención & 3,69 & 0,63 \\
\hline Relevancia & 3,71 & 0,54 \\
\hline Confianza & 3,53 & 0,49 \\
\hline Satisfacción & 3,93 & 0,62 \\
\hline
\end{tabular}

\section{3. Resultados orientación espacial}

En la Tabla 4 se recogen las puntuaciones (porcentaje de respuestas correctas) obtenidas por los alumnos de ambos grupos en cada uno de los sub-test. En la última columna se muestra la puntuación media obtenida por cada alumno en los dos sub-test. Es interesante destacar que los alumnos de ambos grupos (GC y GE) necesitaron los 6 y 5 minutos para completar el sub-test 1 y el sub-test 2, respectivamente. En el caso del post-test, los alumnos del GE completaron los dos sub-test en apenas 3 minutos. Esta mejora en la eficiencia no se produjo en el caso de los alumnos del GC, que usaron el mismo número de minutos que en el pre-test.

\section{Tabla 4}

\begin{tabular}{ccccccc} 
ALUMnO & \multicolumn{2}{c}{ SUB-TEST 1} & \multicolumn{2}{c}{ SUB-TEST 2} & \multicolumn{2}{c}{ MEDIA SUB-TEST } \\
\hline GC & Pre & post & pre & post & pre & post \\
\hline 1 & 10,00 & 40,00 & 16,67 & 50,00 & 13,33 & 45,00 \\
\hline 2 & 40,00 & 20,00 & 0,00 & 0,00 & 20,00 & 10,00 \\
\hline 3 & 30,00 & 20,00 & 0,00 & 0,00 & 15,00 & 10,00 \\
\hline 4 & 20,00 & 20,00 & 0,00 & 16,67 & 10,00 & 18,33 \\
\hline 5 & 50,00 & 40,00 & 0,00 & 50,00 & 25,00 & 45,00 \\
\hline 6 & 60,00 & 40,00 & 66,67 & 33,33 & 63,33 & 36,67 \\
\hline 7 & 40,00 & 40,00 & 0,00 & 33,33 & 20,00 & 36,67 \\
\hline 8 & 40,00 & 50,00 & 16,67 & 0,00 & 28,33 & 25,00 \\
\hline 9 & 30,00 & 70,00 & 16,67 & 50,00 & 23,33 & 60,00 \\
\hline 10 & 20,00 & 20,00 & 50,00 & 0,00 & 35,00 & 10,00 \\
\hline
\end{tabular}




\begin{tabular}{|c|c|c|c|c|c|c|}
\hline 11 & 30,00 & 30,00 & 16,67 & 0,00 & 23,33 & 15,00 \\
\hline 12 & 0,00 & 20,00 & 0,00 & 0,00 & 0,00 & 10,00 \\
\hline 13 & 20,00 & 10,00 & 0,00 & 0,00 & 10,00 & 5,00 \\
\hline 14 & 70,00 & 50,00 & 50,00 & 50,00 & 60,00 & 50,00 \\
\hline 15 & 30,00 & 30,00 & 16,67 & 0,00 & 23,33 & 15,00 \\
\hline 16 & 60,00 & 50,00 & 33,33 & 66,67 & 46,67 & 58,33 \\
\hline 17 & 30,00 & 50,00 & 100,00 & 50,00 & 65,00 & 50,00 \\
\hline 18 & 60,00 & 70,00 & 16,67 & 33,33 & 38,33 & 51,67 \\
\hline 19 & 20,00 & 40,00 & 16,67 & 50,00 & 18,33 & 45,00 \\
\hline 20 & 60,00 & 80,00 & 66,67 & 50,00 & 63,33 & 65,00 \\
\hline 21 & 20,00 & 50,00 & 0,00 & 16,67 & 10,00 & 33,33 \\
\hline \multicolumn{7}{|l|}{ GE } \\
\hline 1 & 40,00 & 50,00 & 0,00 & 16,67 & 20,00 & 33,33 \\
\hline 2 & 10,00 & 20,00 & 0,00 & 0,00 & 5,00 & 10,00 \\
\hline 3 & 40,00 & 50,00 & 66,67 & 83,33 & 53,33 & 66,67 \\
\hline 4 & 50,00 & 70,00 & 50,00 & 50,00 & 50,00 & 60,00 \\
\hline 5 & 30,00 & 40,00 & 16,67 & 16,67 & 23,33 & 28,33 \\
\hline 6 & 50,00 & 50,00 & 16,67 & 33,33 & 33,33 & 41,67 \\
\hline 7 & 40,00 & 40,00 & 16,67 & 16,67 & 28,33 & 28,33 \\
\hline 8 & 70,00 & 40,00 & 0,00 & 33,33 & 35,00 & 36,67 \\
\hline 9 & 40,00 & 60,00 & 66,67 & 83,33 & 53,33 & 71,67 \\
\hline 10 & 30,00 & 60,00 & 33,33 & 0,00 & 31,67 & 30,00 \\
\hline 11 & 10,00 & 10,00 & 0,00 & 0,00 & 5,00 & 5,00 \\
\hline 12 & 60,00 & 50,00 & 0,00 & 16,67 & 30,00 & 33,33 \\
\hline 13 & 60,00 & 50,00 & 33,33 & 16,67 & 46,67 & 33,33 \\
\hline 14 & 40,00 & 40,00 & 16,67 & 33,33 & 28,33 & 36,67 \\
\hline 15 & 20,00 & 60,00 & 50,00 & 16,67 & 35,00 & 38,33 \\
\hline 16 & 30,00 & 0,00 & 0,00 & 0,00 & 15,00 & 0,00 \\
\hline 17 & 30,00 & 30,00 & 16,67 & 66,67 & 23,33 & 48,33 \\
\hline 18 & 40,00 & 20,00 & 0,00 & 16,67 & 20,00 & 18,33 \\
\hline 19 & 20,00 & 40,00 & 16,67 & 33,33 & 18,33 & 36,67 \\
\hline 20 & 10,00 & 30,00 & 33,33 & 50,00 & 21,67 & 40,00 \\
\hline 21 & 50,00 & 60,00 & 66,67 & 66,67 & 58,33 & 63,33 \\
\hline 22 & 30,00 & 40,00 & 50,00 & 50,00 & 40,00 & 45,00 \\
\hline 23 & 60,00 & 60,00 & 0,00 & 50,00 & 30,00 & 55,00 \\
\hline 24 & 10,00 & 10,00 & 0,00 & 0,00 & 5,00 & 5,00 \\
\hline
\end{tabular}


Los resultados que se presentan en la Tabla 4 muestran que en el sub-test 1, el 42\% del GC mejora su puntuación (9 de 21 alumnos), el 24\% se queda igual (5 de 21) y el 33\% empeora el resultado (7 de 21). En el caso del GE, el 50\% de los alumnos ha mejorado su puntuación (12 de 24 alumnos), el 30\% (7 de 24 alumnos) ha obtenido la misma puntuación en ambos test y el $20 \%$ ha empeorado el resultado ( 5 de los 24 alumnos). Así, se observa que los resultados han sido mejores en el caso del GE.

La mejora de los resultados del GE es más clara en el sub-test 2. En el caso del GC, el 42\% de los alumnos mejoran su puntuación en el post-test, el 24\% mantiene la nota, mientras que el 33\% empeora su puntuación. En el GE, los alumnos que mejoran su puntuación alcanzan el 50\% del total, el 37.5\% se queda igual, mientas que sólo el 12.5\% obtiene una puntuación menor en el post-test.

La Figura 9 muestra la media de la puntuación obtenida por cada alumno en ambos sub-test. Se usa la representación poligonal explicada anteriormente. Usando esta representación de los resultados también se observa una mejoría más notable de las puntuaciones del post-test en el caso del GE.

\section{Figura 9}

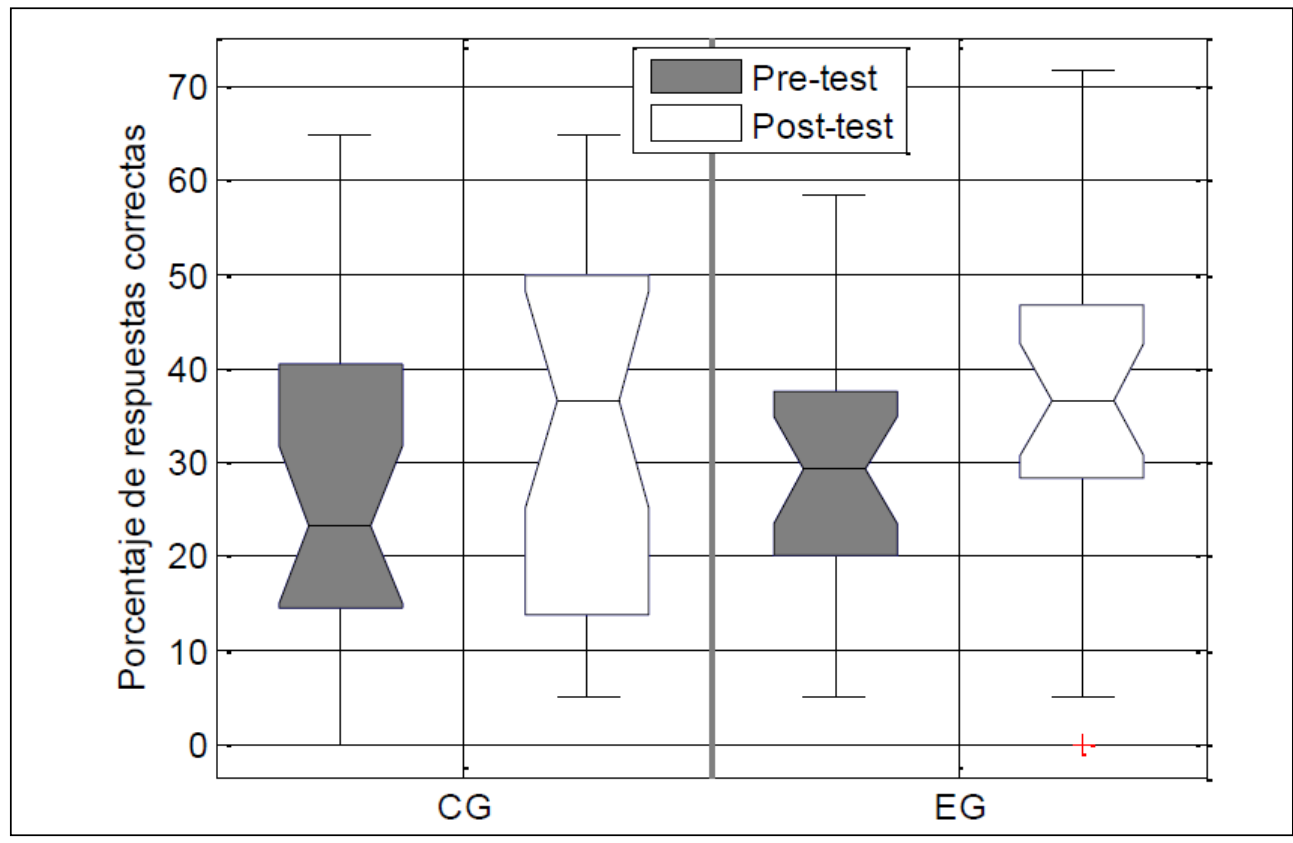


Finalmente, con el objetivo de comparar el GC y el GE de forma global, en la Tabla 5 se muestra la media y la desviación estándar de las puntuaciones obtenidas por parte de cada grupo en los dos sub-test. Además, se ha realizado un t-test para comparar las medias obtenidas en cada pre-test y post-test. Específicamente, se ha realizado una prueba de dos colas (two-tailed test), tomando $\alpha=0.05$ como nivel de significancia (5\%).

\section{Tabla 5}

Puntuaciones obtenidas en pre y en el post-test (GC y GE) (media y desviación estándar de todo el grupo)

\begin{tabular}{|c|c|c|c|c|c|c|c|c|c|c|c|c|}
\hline \multirow{3}{*}{ Test } & \multicolumn{6}{|c|}{ GC } & \multicolumn{6}{|c|}{ GE } \\
\hline & \multicolumn{2}{|c|}{ media (\%) } & \multicolumn{2}{|c|}{ SD (\%) } & \multirow{2}{*}{$\mathrm{t}$} & \multirow{2}{*}{$\mathrm{P}$} & \multicolumn{2}{|c|}{ Media (\%) } & \multicolumn{2}{|c|}{ SD (\%) } & \multirow{2}{*}{$\mathrm{t}$} & \multirow{2}{*}{$\mathrm{p}$} \\
\hline & Pre & Post & pre & post & & & Pre & Post & Pre & Post & & \\
\hline 1 & 35,24 & 40,00 & 18,87 & 18,70 & $-0,82$ & 0,42 & 36,25 & 40,83 & 17,39 & 18,39 & $-0,89$ & 0,38 \\
\hline 2 & 23,02 & 26,19 & 28,12 & 23,90 & $-0,39$ & 0,69 & 22,92 & 31,25 & 23,98 & 26,15 & $-1,15$ & 0,25 \\
\hline Media & 29,1 & 33,1 & 24,45 & 22,32 & $-0,78$ & 0,44 & 29,6 & 36 & 21,79 & 22,88 & $-1,41$ & 0,16 \\
\hline
\end{tabular}

Como se observa en la Tabla 5, la media de las notas en cada grupo en el caso del sub-test 1 se obtiene parecida en el GC y en el GE. Concretamente, se pasa de un porcentaje de respuestas correctas de $35.24 \%$ en el pre-test a un $40 \%$ en el post-test en el caso del GC, mientras que en el caso del GE se pasa de un $36.25 \%$ a un $40.83 \%$. En el caso del sub-test 2, la mejora en el caso del GE es claramente más notable: se pasa de un porcentaje de respuestas correctas de $22.92 \%$ a un $31.25 \%$, mientras que en caso del GC se pasa de un $23.02 \%$ a un $26.19 \%$.

Aunque la mejora de la media obtenida en el post-test no es estadísticamente significativa en ninguno de los casos (nótese que el valor de p siempre es mayor que 0.05), se puede observar que en el caso del GE, se obtienen valores menores de p que en el GC. Concretamente, el GE obtiene $\mathrm{p}=0.38$ y 0.25 en el sub-test 1 y sub-test 2 , respectivamente, mientras que en el caso de GC p $=0.42$ y 0.69 en los mismos sub-test.

Si consideramos la media que se obtiene en ambos test (última fila de la Tabla 5), se observa que la mejora es más destacable en el caso del GE. En particular, se pasa de un 29.13\% a un 33.10\% en el caso del GC y de un $29.58 \%$ a un 36.04\% en el caso del GE. De nuevo, el valor de p es menor en el caso de GE que en GC (0.16 y 0.44, respectivamente). Si bien el valor de p es no es menor que 0.05 , en el caso del GE está cerca de serlo. Es decir, que la diferencia entre la media obtenida en el pre-test y en la media obtenida en el post-test está cerca de ser estadísticamente significativa. En el caso del GC, la diferencia entre ambas medias está lejos de ser menor que 0.05.

Hay que tener en cuenta que los alumnos no habían visto ni usado test similares antes ni habían estado entrenados para ello. Además, tal y como se mencionó anteriormente, estos test son más adecuados para edades más avanzadas. Posiblemente unos test más adaptados a la edad de la muestra permitirían visualizar mejor la mejora en las habilidades espaciales. Por otro lado, un mayor número de sesiones prácticas también ayudaría a que mejora de los resultados fuera estadísticamente significativa. Es importante destacar que los alumnos que tienen una orientación espacial muy poco desarrollada mejorarán esta habilidad muy lentamente durante las primeras 6 sesiones, aproximadamente (Newcombe, 2010). 


\section{CONCLUSIONES}

El principal objetivo de esta investigación era el de potenciar la orientación espacial a través de la Educación Física. Para ello, se ha diseñado una secuencia didáctica para $5^{\circ}$ de Primaria siguiendo dos estilos de enseñanza-aprendizaje altamente competenciales: el estilo de producción convergente y el estilo de producción divergente, organizando a los alumnos en grupos de 4/5. Dicho estilo y organización ha facilitado la adquisición de contenidos actitudinales: trabajo en equipo, cooperación y respeto y esfuerzo y superación, entre otros.

El hecho de trabajar en grupo compartiendo decisiones y proponiendo soluciones mediante la escucha y el diálogo, pero al mismo tiempo realizando actividades partiendo de la autonomía e iniciativa personal ha facilitado que el alumnado y el docente interioricen la conveniencia de la cooperatividad y la responsabilidad compartida en los distintos procesos para alcanzar un aprendizaje competencial.

Para evaluar la mejora de los alumnos en cuanto a orientación espacial, se consideró un grupo experimental (GE), que asistió a las sesiones de Educación Física, y un grupo control (GC), que permitió validar los instrumentos de control. Se diseñaron un pre-test y un post-test para evaluar la orientación espacial de los alumnos antes y después de participar de la secuencia didáctica. Los resultados obtenidos evidencian una mejora más notable de la orientación espacial en el caso de los alumnos del GE. De todos modos, la mejora no ha sido significativa. Como se ha comentado en el apartado anterior, un número más elevado de sesiones ayudaría posiblemente a obtener una mejora más significativa de la orientación espacial por parte de los alumnos del GE. También se ha comentado anteriormente que los tests fueron diseñados para estudiantes de mayor edad y no se ha realizado ninguna adaptación para adecuarlos a los estudiantes del presente trabajo. Por otro lado, se evaluó de forma cuantitativa el grado de motivación de los alumnos del GE durante las sesiones de Educación Física. Las puntuaciones obtenidas en el test de motivación muestran el alto grado de motivación por parte de los alumnos de este grupo. Finalmente, después de cada sesión práctica, se pasó una ficha de autoevaluación que demuestra el alto grado de implicación del alumnado, especialmente en las actividades cooperativas, y el nivel de exigencia personal, muchas veces superior al de los propios docentes en la heteroevaluación.

Así pues, se confirma que la Educación Física permite trabajar la orientación espacial de una forma lúdica y motivadora. En clase de matemáticas y siguiendo una metodología tradicional es difícil trabajar ésta y otras de las habilidades espaciales.

Además de la orientación espacial, durante las sesiones prácticas, los alumnos estuvieron usando el lenguaje espacial a la hora de describir e interpretar recorridos. En este trabajo no se ha cuantificado la evolución de los alumnos con respeto a esta habilidad de comunicación, pero realmente se ha observado una mejora en las capacidades comunicativas. Destacar también que se ha trabajado interpretación de mapas, planificación y diseño de los mismos, lectura de recorridos en distintos soportes (gráfico, textual...) y realizados por diferentes autores (maestro de Educación Física, compañeros de equipo o contrincantes). La finalidad de las diferentes actividades siempre ha sido interpretar una información, resolver la incógnita o problema planteado y encontrar una solución o varias para el mismo, partiendo siempre del trabajo cooperativo, del protagonismo del alumno y de la responsabilidad compartida para conseguir un objetivo común.

Se puede concluir que usando una propuesta similar se podrían trabajar contenidos matemáticos o de otras áreas experimentales de una forma competencial y a la vez más motivadora para los alumnos. 


\subsection{Implicaciones didácticas}

Concluido el estudio debemos destacar la necesidad de potenciar el uso de metodologías de producción en el aula. Los alumnos están más motivados y aprenden de forma distinta cuando son los protagonistas de sus aprendizajes. Si partimos de una situación problema les estamos obligando a utilizar todas sus competencias para resolverla de manera eficiente y aunque estamos trabajando contenidos concretos de cualquier área curricular lo estamos haciendo partiendo de la globalidad de la enseñanza.

Es importante también comentar que este tipo de sesiones de Educación Física requieren planificación y preparación previa por parte del especialista, es decir, tiempo, y no siempre se dispone. Una buena organización y, en caso que se considere oportuno, la participación o ayuda del alumnado en el proceso, así como una alta motivación en el acto didáctico permitirán mejorar nuestra calidad docente.

En un mundo cada vez más global, la interdisciplinariedad es un concepto clave y factible, solo es cuestión de proponer una adaptación metodológica a nivel de centro e ir animando a los compañeros docentes a dar el paso.

\section{BIBLIOGRAFÍA}

Bakker, M. (2008). Spatial ability in primary school: Effects of the tridio learning material. Master Thesis of Psychology. University of Twente, Enschede.

Blázquez, D. (2013). Diez competencias docentes para ser mejor profesor de Educación Física: la gestión didáctica de la clase. Barcelona: INDE.

Bocanegra, C. y Villanueva, A. (2003). Pautas para la elaboración de mapas de orientación de centros escolares y de jardines. Retos, 6, 21-25.

Díaz, J. (2010). El desarrollo de la competencia matemática desde la Educación Física. Aula de Innovación Educativa, 189, 23-29.

Hegarty, M. y Waller, D. (2004). A dissociation between mental rotation and perspective-taking spatial abilities. Intelligence, 32, 175-191.

Julià, C. y Antolí, O. (2016). Spatial ability learning through educational robotics. International Journal of Technology and Design Education, 26 (2), 185-203.

Keller, J. M. (1987). Development and use of the ARCS model of instructional design. Journal Instructional Development, 10 (3), 2-10.

Keller, J. M. (2006). Development of Two Measures of Learner Motivation (draft). Tallahasse: Florida State University.

NCTM (2000). Principles and standards for school mathematics. Reston, VA: National Council of Teachers of Mathematics (NCTM).

Newcombe, N. (2010). Picture this. Increasing Math and Science Learning by Improving Spatial Thinking. American Educator, 29-43. 
Sallan, C. (2002). Orientación a pie y en bicicleta. Un medio para trabajar la orientación en el medio natural y al mismo tiempo promocionar la utilización de la bicicleta en la ESO. Apunts. Educación Física y Deportes, 69, 96-103.

Sebastiani, E.M. (2003). Reptes de l’Educació Física del S. XXI. Revista de psicología, ciencias de la Educación Física $y$ del deporte Blanquerna, 12.122-129.

Sorby, S.A. (2009). Educational research in developing 3-D spatial skills for engineering students. International Journal of Science Education, 31 (3), 459-480.

Sutton, K., Williams A. P. (2007). Spatial cognition and its implications for design, en Proceedings of IASDR'07: International Association of Societies of Design Research 2007. Hong Kong (China).

Sutton, K., Heathcote, A. y Bore, M. (2005). Implementing a Web-based measurement of 3-D understanding. Proceedings of the 19th conference of the Computer-Human Interaction Special Interest Group (CHISIG) of Australia on computer-human interaction: Citizens online; considerations for today and the future (pp. 1-4). Narrabundah (Australia): CHISIG.

Valero, V., Granero, A., Gómez, M., Padilla, F. y Gutiérrez, H. (2010). Diferentes propuestas para la enseñanza de la orientación a nivel escolar: orientación en el aula de Educación Física, orientación urbana y orientación subacuática. Apunts. Educación Física y Deportes, 99, 34-46.

Verner, I. (2004). Robot manipulations: A synergy of visualization, computation and action for spatial instruction. International Journal of Computers for Mathematical Learning, 9, 213-234.

Zabala, A. (2014). Métodos para la enseñanza de las competencias. Barcelona: Graó.

NOTAS

${ }^{1}$ http://www.spatiallearning.org

\section{INFORMACIÓN SOBRE LOS AUTORES}

Míriam Segura. Maestra de Educación Primaria especialista en Educación Física, Licenciada en Ciencias de la Actividad Física y el Deporte por la Universidad Ramon Llull (2006) y doctora en Pedagogía por la Universidad Rovira i Virgili (2013). Desde 2009 forma parte del personal docente e investigador del Departamento de Pedagogía de la URV. Actualmente es profesora en comisión de servicios en el Campus Tierras del Ebro e imparte clases de Educación Física y Psicomotricidad en los Grados de Educación Primaria y Educación Infantil. Su investigación se centra en la didáctica de estas áreas y en las actividades físicas en la educación no formal. 
Carme Julià. Licenciada en Matemáticas por la Universidad Politécnica de Cataluña (2002) y doctora en Informática por la Universidad Autónoma de Barcelona (2008). Desde 2008 forma parte del personal docente e investigador del Departamento de Ingeniería Informática y Matemáticas, en la Universidad Rovira y Virgili. Actualmente forma parte del grupo de investigación Didáctica de las Matemáticas y es profesora agregada en el DEIM. Su docencia está centrada en la Enseñanza y Aprendizaje de las Matemáticas en los Grados de Educación Primaria y Educación Infantil. Su investigación se centra en la didáctica de las Matemáticas.

$\triangle$ carme.julia@urv.cat 